\title{
DYNAMIC ASPECTS OF METARHODOPSIN FORMATION IN PHOTOCHEMICAL CYCLE OF EXTRACTED OCTOPUS RHODOPSIN
}

\author{
Yoshio Ebina, Nobukata Nagasawa*, and Yasuo Tsukahara** \\ Research Institute of Electrical Communication, Tohoku University \\ *Department of Physics, Fuculty of Science, Tohoku University \\ **Department of Physiology, Tohoku University School of Medicine, Sendai
}

\begin{abstract}
Summary Changes in the difference spectrum of the octopus rhodopsin solution with $\mathrm{pH} 10.3$ were studied at about $2^{\circ} \mathrm{C}$ by applying the flash photolytic technique. The main peak (about $520 \mathrm{~nm}$ ) in the difference spectrum at $10 \mathrm{msec}$ after a flash coincided with the peak of the calculated difference spectrum between rhodopsin and acid metarhodopsin. It was suggested that in the octopus rhodopsin cycle the acid metarhodopsin may be a precursor of the alkaline metarhodopsin even in alkaline solution.
\end{abstract}

The vertebrate rhodopsin bleaches after irradiation of light via a series of intermediates (bathorhodopsin, lumirhodopsin, metarhodopsin I, II, and pararhodopsin) to the final products, retinal and opsin (see YoshizawA, 1972). On the other hand, a number of invertebrate rhodopsins are photoconverted in the light to metarhodopsins, which release retinal only very slowly (see GoLDSMITH, 1972). A certain physiologically active intermediate has been reported to play an important role in photoexcitation in the invertebrate visual cell. Nolte and BRown (1972) observed in the UV-sensitive visual cell of Limulus median ocellus that the photoreceptor membrane is depolarized by UV illumination and the UVelicited depolarization can be repolarized by a superposed visible light of long wavelength, and that the spectral sensitivity curve of the repolarizing response peaks at $480 \mathrm{~nm}$. From these findings they introduced a hypothesis that the photoinduced reversal change in the membrane potential was the results of the photointerconversion between the UV-sensitive rhodopsin and a thermally stable intermediate (M 480).

HAMDORF and SCHWEMER (1971) have revealed in their studies on the UV cells of the Ascalaphus macaronius that an intermediate which has absorption

Received for publication November 12, 1973

蛕名良雄, 長沢信方, 塚原保夫 
maximum at around $480 \mathrm{~nm}$ is an acid form of metarhodopsin. Then it may be considered that the active intermediate (M 480) is also acid form of metarhodopsin and that, at least in some invertebrate visual cells, the essential processes for generation and recovery of the photoreceptor potential may couple with the photo-interconversion between rhodopsin and acid metarhodopsin.

The chemical characteristics of the metarhodopsins in invertebrate rhodopsin cycle have been most extensively studied in the cephalopod. Both of two forms of metarhodopsin, acid and alkaline metarhodopsins, are stable in solution below $20^{\circ} \mathrm{C}$ (Hubbard and ST. George, 1958; Kropf et al., 1959; Yoshizawa and Wald, 1964). These intermediates have been believed to be in a thermal tautomeric equilibrium which depends upon the temperature, $\mathrm{pH}$ and ionic composition of the solution (MATTHEWs et al., 1963).

In the present experiment the transient changes in optical absorption spectrum of the octopus rhodopsin solution were observed by the technique of flash-photolysis in order to study the process of the formation of acid metarhodopsin at around $2{ }^{\circ} \mathrm{C}$. It was found that acid metarhodopsin is photoconverted from rhodopsin independently of $\mathrm{pH}$ of the solution. Some of the converted acid metarhodopsins are then transformed to metarhodopsin of alkaline form to reach a tautomeric equilibrium which is decided by $\mathrm{pH}$ of the solution.

\section{MATERIALS AND METHODS}

Large-eyed octopuses were used for the experiments. The animals (species not yet identified and locally called as amadako) were captured in the sea off the coast of Fukushima Prefecture in the northeast district of Japan. The absorption maxima of rhodopsin, acid metarhodopsin and alkaline metarhodopsin are 470, 495, and $370 \mathrm{~nm}$, respectively (Suzuki, T., personal communication).

Rhodopsin was extracted from eyes which were enucleated from darkadapted animals. Omochrome and salts were removed by passing the crude rhodopsin solution ( $2 \%$ digitonin in phosphate buffer, $\mathrm{pH} 6.1$ ) through DEAE and Sephadix G-25 columns (ERHARDT et al., 1966; SUZUKI et al., 1972). The solution was freeze dried in the presence of dim red light and stored in a freezer. Before each experiment, the rhodopsin solution was prepared by following ways. The stored sample $(50$ or $70 \mathrm{mg}$ ) was dissolved in the $10 \mathrm{ml}$ of phosphate buffer at $\mathrm{pH}$ 6.1. The $\mathrm{pH}$ of the solution was then increased to 10.3 by adding some grains of $\mathrm{Na}_{2} \mathrm{CO}_{3}$ to phosphate buffer. The $\mathrm{pH}$ of the solution was recorded for observing the change in $\mathrm{pH}$ following photoreactions of rhodopsin, and the change was less than 0.1 . The temperature was monitored by a thermister immersed in the solution.

The schematic diagram of the optical arrangement and the recording system is represented in Fig. 1. The rhodopsin solution in a sample cell $\left(\mathrm{Cl} ; 2 \times 0.8 \times 2 \mathrm{~cm}^{3}\right)$ was cooled down to about $2^{\circ} \mathrm{C}$ by steeping the cell in ice cold water in a trans- 


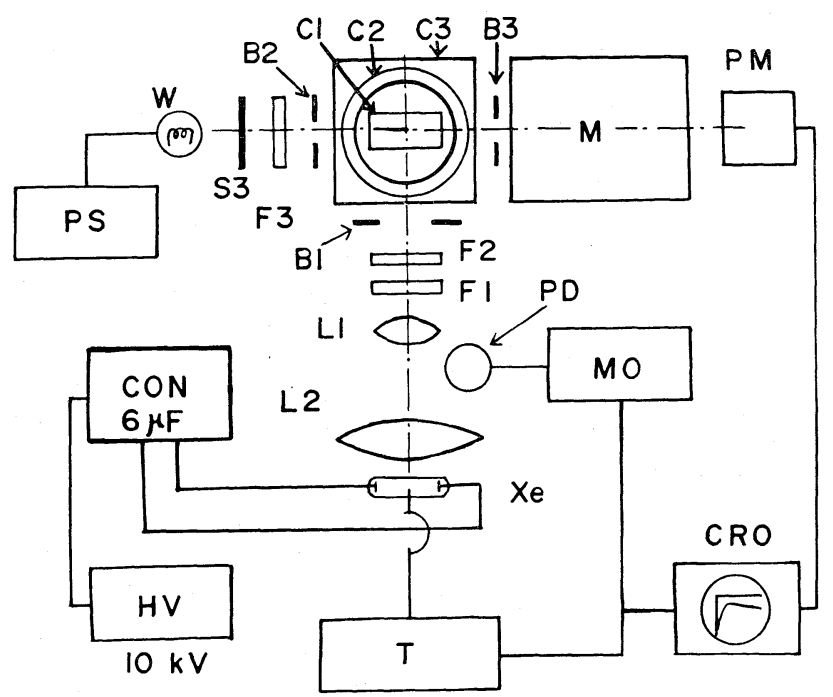

Fig. 1. Schematic diagram of the apparatus. $\mathrm{C} 1$ : sample cell $\left(2 \times 0.8 \times 2 \mathrm{~cm}^{3}\right), \mathrm{C} 2$ : dewar, $\mathrm{C} 3$ : water cell, Xe: xenon flash lamp for exciting light, CON: oil condenser bank $(6 \mu \mathrm{F})$, HV: high voltage power supply for Xe, L1, L2: condenser lenses, F1: yellow glass filter (Toshiba VY-46), F2: heat filter, F3: interference filter, M: monochromator (band pass; $5.6 \mathrm{~nm}$ ), PM: photomultiplier (HTV R-106), W: tungsten lamp for measuring beam $(40 \mathrm{~W})$, PS: regulated power supply for $\mathrm{W}, \mathrm{B} 1, \mathrm{~B} 2$, B3: light baffles, PD: photodiode, MO: monitoring system for intensity of exciting light, CRO: oscilloscope, T: trigger circuit, $\mathrm{S} 3$ : shutter.

parent dewar (C2) flask. The dewar flask was placed in a cubic glass cell (C3) filled with water to avoid the refraction of the measuring beam from the round surface of the dewar flask.

A light beam from a xenon lamp (Xe; 300 joules, with half duration of about $300 \mu \mathrm{sec}$ ) was used as an exciting light. The beam was focussed on a sample cell (C1) through a set of condenser lenses (L1, L2), a heat filter (F2) and a yellow glass filter (F1; Toshiba VY-46). For measuring the intensity of the transmitting light through the sample cell, another light beam from a tungsten lamp (W) was set at a right angle to the axis of the exciting beam. An interference filter for a selected wavelength (F3) was placed in front of the sample cell, and a shutter (S3) was opened only during the measurement to minimize the photoreaction by the measuring beam. A Bauch and Lomb grating monochromator ( $\mathrm{M}$; band pass, $5.6 \mathrm{~nm}$ ) was interposed between the sample cell and photomultiplier (PM; HTV-R 106) The wavelength of the measuring light was changed by exchanging the interference filter and by adjusting the wavelength of the monochromator.

Stray light from the exciting flash was minimized by light baffles (B1, B2, B3). The intensity of each flash was monitored by a photodiode (PD) and the fluctua- 
tion of light was found to be within $5 \%$. The output signals from the photomultiplier were displayed on an oscilloscope (CRO) and photographically recorded. The xenon flash, the oscilloscope and a monitoring system (MO) were triggered by the same pulse from a trigger circuit $(\mathrm{T})$.

\section{RESULTS AND DISCUSSION}

In order to study the transient change in the density spectrum of the rhodopsin solution, the change in intensity of the transmitting light through a sample was recorded after an exciting flash at several wavelengths. Figure 2 shows examples of the transient change in intensity of the transmitting light at $380 \mathrm{~nm}, 490 \mathrm{~nm}$, and $560 \mathrm{~nm}$ through the octopus rhodopsin solution at $\mathrm{pH} 10.3$.

A monotonous decrease in intensity of the measuring light with time after a flash (increase in absorption) was observed at $380 \mathrm{~nm}$ (Fig. 2A). This change in alkaline solution may be attributed mainly to the transformation of alkaline metarhodopsin which has an absorption maximum at $370 \mathrm{~nm}$. At wavelengths longer than $450 \mathrm{~nm}$, a monotonous decrease in absorption was preceded by a transient increase in absorption of the measuring light (Fig. 2B and Fig. 2C). The transient increase peaked at about $10 \mathrm{msec}$ after the flash. These results would suggest that the rhodopsin is converted to alkaline metarhodopsin via an intermediate with a rather long lifetime and a larger extinction coefficient at around $500 \mathrm{~nm}$ than that of rhodopsin.
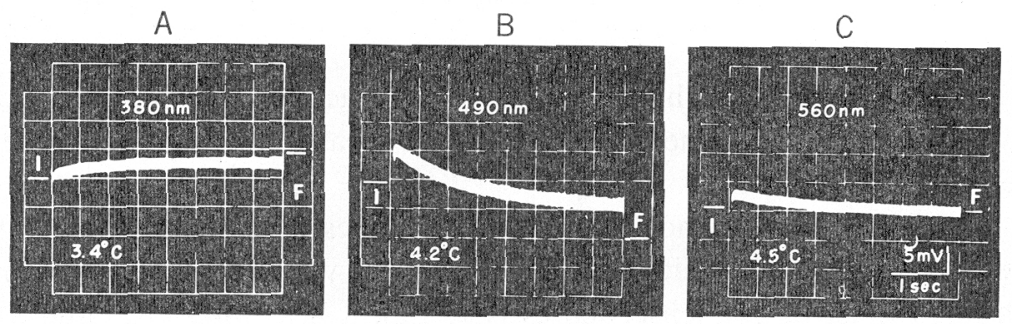

Fig. 2. Time course of changes in light transmission at $380 \mathrm{~nm}, 490 \mathrm{~nm}$, and $560 \mathrm{~nm}$ through photoexcited rhodopsin solution $(\mathrm{pH} ; 10.3)$ following an exciting flash. Traces are read from left to right. A downward deflection indicates increased transmission, and upward deflection indicates decreased transmission. Horizontal bars in each picture show initial (I) and final (F) levels of transmission, respectively. Relative intensity of exciting light, A, $1.00 ; \mathrm{B}, 1.01 ; \mathrm{C}, 0.99$.

To identify the intermediate in question, the difference spectra were obtained by measuring the change in optical density $(\angle \mathrm{OD})$ before and after the illumination. The $\triangle \mathrm{OD}$ at each wavelength was calculated by subtracting $\log \mathrm{I}(t)$ from $\log \mathrm{I}(t=0)$, where $\mathrm{I}(t=0)$ and $\mathrm{I}(t)$ represent the intensities of the transmitting light before and $t \mathrm{sec}$ after the flash, respectively. 

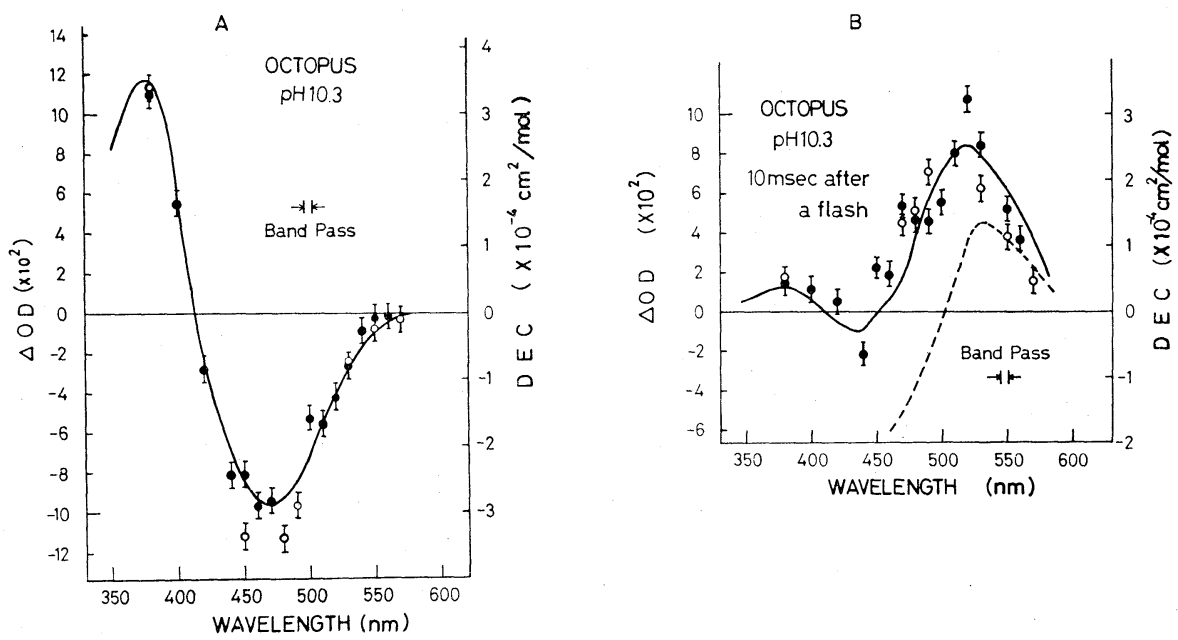

Fig. 3. Difference absorption spectra. A: Points marked by $(0.5$ wt. $\%)$ and by $\bigcirc$ $(0.7 \mathrm{wt} . \%)$ indicate change in optical density ( $\triangle \mathrm{OD})$ during $100 \mathrm{sec}$ after a flash. Vertical bar attached to each point shows the range of experimental error. Solid curve represents difference spectrum (DEC) between rhodopsin and alkaline metarhodopsin. Left ordinate indicates optical density for $\triangle \mathrm{OD}$ and right ordinate indicates extinction coefficient for DEC. DEC/ $\triangle O D$ is $3 \times 10^{6} \mathrm{~cm}^{2} / \mathrm{mol}$. B: Each point indicates $\triangle O D$ during $10 \mathrm{msec}$ after a flash. Solid curve is DEC between rhodopsin and acid metarhodopsin. Dashed curve shows DEC between rhodopsin and lumirhodopsin of Octopus vulgaris.

Figure $3 \mathrm{~A}$ shows $\triangle \mathrm{OD}$ during $100 \mathrm{sec}$ after the flash as a function of the wavelength. Filled and open circles were obtained at the concentration of 0.5 and $0.7 \mathrm{wt} . \%$, respectively. A vertical bar attached to each circle shows the range of the experimental error mainly due to the fluctuations of the signal in the measuring system. These plots indicate an increase in optical density at $380 \mathrm{~nm}$ and a decrease at around $480 \mathrm{~nm}$ in alkaline solution during $100 \mathrm{sec}$ after the flash. Then the difference spectrum in optical density was compared with the difference spectrum between rhodopsin and alkaline metarhodopsin. The latter spectrum was calculated from the difference in extinction coefficient (will be called hereafter DEC). The computed DEC was superposed by a solid curve in Fig. 3A. The scale for the DEC was drawn in the right ordinate so as to match both spectra at $510 \mathrm{~nm}$. General shapes of the spectrum in $\triangle \mathrm{OD}$ and that in DEC are in substantial agreement with each other. This agreement may manifest the formation of alkaline metarhodopsin as a stable product in the alkaline solution as reported by static experiments (HuBBARD and ST. GEORGE, 1958).

Filled and open circles in Fig. 3B show the $\triangle$ OD's for $10 \mathrm{msec}$ after the exciting flash. In this figure the plots exhibit a predominant increase in the optical density at around $520 \mathrm{~nm}$. It was suggested from this observation that the flash 
illumination developes an intermediate which has a higher extinction coefficient than that of rhodopsin at around $520 \mathrm{~nm}$ during $10 \mathrm{msec}$ after the flash.

The DEC between rhodopsin and acid metarhodopsin of amadako was superposed in Fig. 3B by a solid curve. As shown in the figure, the plots for $\triangle \mathrm{OD}$ are distributed on the curve through whole wavelengths. Although it seems clear that the transient increase in absorption at around $520 \mathrm{~nm}$ (see Fig. 2B, C) is the result of the acid metarhodopsin formation even in alkaline solution, another possibility should be considered before drawing this conclusion.

If an intermediate which is transformed prior to the metarhodopsins in the photochemical cycle has a similar absorption spectrum to that of acid metarhodopsin, the same result may be obtained by the formation of this intermediate. The possible intermediates that have been reported are bathorhodopsin and lumirhodopsin (YosHIZaWA and WALD, 1964).

The decay time of the bathorhodopsin of cattle at room temperature is about $50 \mathrm{nsec}$ (ROSENFELD et al., 1972; BusCH et al., 1972), and the squid bathorhodopsin has been suggested to have a narrower stability range than that of cattle bathorhodopsin (YoshizAwA and WALD, 1964). Then it is assumed that the bathorhodopsin formation could not be detected in the present experiments because of far larger time resolution of the measuring system than the decay time.

As for the octopod lumirhodopsin, only the absorption spectrum of this intermediate of Octopus vulgaris has been reported (KROPF et al., 1959), and there is no available information about the absorption spectrum of the intermediate of amadako. The DEC between rhodopsin and lumirhodopsin of Octopus vulgaris was represented by a dashed curve in Fig. 3B for reference. The shape of the dashed curve is different from that of the solid curve especially at the shorter wavelengths as shown in the figure.

The absorption spectrum of lumirhodopsin of Octopus vulgaris may be somewhat different from that of amadako, and the dashed curve for Octopus vulgaris would be inadequate for a precise comparison. However, the precursor of lumirhodopsin (bathorhodopsin) should be unstable at around $2^{\circ} \mathrm{C}$ as described earlier, and the time course of the lumirhodopsin formation may be very rapid in the octopus rhodopsin cycle comparing with the rise time of the transient increase in absorption which is shown in Fig. $2 \mathrm{~B}$ (more than $10 \mathrm{msec}$ ). Then, the possibility that the transient increase at around $520 \mathrm{~nm}$ is due to the formation of lumirhodopsin would be safely excluded. Therefore it seems plausible that the transient increase in absorption at around $520 \mathrm{~nm}$ is caused by the formation of acid metarhodopsin even in alkaline solution.

Assuming that the acid metarhodopsin is transformed antecedently to the alkaline metarhodopsin in alkaline solution, one might expect that the slow decrease in absorption following the transient increase is greatly affected by decreasing the $\mathrm{pH}$ of the solution as a result of the accumulation of acid metarhodopsin. In fact, as shown in Fig. 4, the time course of the change in absorp- 


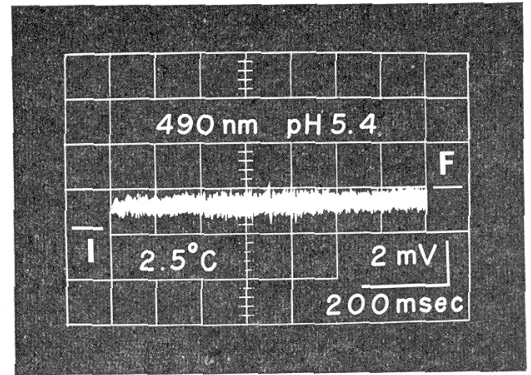

Fig. 4. Time course of changes in light transmission through photoexcited rhodopsin solution at pH 5.4. Trace is read from left to right. An upward deflection indicates decreased transmission. Horizontal bars show initial (I) and final (F) levels of transmission respectively.

tion of acid solution is different from that of alkaline solution (see Fig. 2B). The trace in Fig. 4 indicates the suppression of the alkaline metarhodopsin formation.

On the basis of these findings, following conclusion may be made. The acid metarhodopsin is photoconverted from rhodopsin independently of the $\mathrm{pH}$ of the solution. In alkaline solution, the acid metarhodopsin which is transformed from rhodopsin at early stages of the photochemical reactions is converted to the alkaline metarhodopsin during $100 \mathrm{sec}$ after the flash. In other words, the acid metarhodopsin is a precursor of the alkaline metarhodopsin even in alkaline solution.

Recently Tsukahara et al. (manuscript in preparation) have observed, in their studies on the early receptor potential of isolated octopus retina, that the acid metarhodopsin is photoconverted from rhodopsin independently of the $\mathrm{pH}$ of the perfusing solution. They speculated that at least in the viable retina the stable photoproduct of rhodopsin may be primarily acid form of metarhodopsin. This view may be consistent with the present conclusion.

The authors express their thanks to Professor N. Saito, Professor M. Ueta, and Professor K. Tasaki, of Tohoku University, for their constant interest and encouragement. Their thanks are also to Mr. S. Aizawa for his assistance in carrying out the experiment. This work was partly supported by a grant-in-aid from the Ministry of Education.

\section{REFERENCES}

Busch, G. E., Applebury, M. L., Lamola, A. A., and Rentzepis, P. M. (1972) Formation and decay of prelumirhodopsin at room temperatures. Proc. Nat. Acad. Sci. USA, 69: 2802-2806.

Erhardt, F., Ostroy, S. E., and Abrahamson, E. W. (1966) Protein configuration in the photolysis of rhodopsin-I. The thermal decay of cattle lumirhodopsin in vitro-. Biochim. Biophys. Acta, 112: 256-264.

Goldsmith, T. H. (1972) The natural history of invertebrate visual pigments. In Handbook 
of Sensory Physiology, ed. by Dartnall, H. J. A. Springer-Verlag, Berlin, Heidelberg, and New York, Vol. 7/1, pp. 685-719.

Hamdorf, K. and Schwemer, J. (1971) Insect visual pigment sensitive to ultraviolet light. Nature, 231: 485-459.

Hubbard, R. and St. George, R. C. C. (1958) The rhodopsin system of the squid. J. Gen. Physiol., 41: 501-528

Kropf, A., Brown, P. K., and Hubbard, R. (1959) Lumi- and metarhodopsin of squid and octopus. Nature, 133: 446-448.

Matthews, R. G., Hubbard, R., Brown, P. K., and Wald, G. (1963) Tautomeric forms of metarhodopsin. J. Gen. Physiol., 47: 215-240.

Nolte, J. and Brown, J. E. (1972) Ultraviolet-induced sensitivity to visible light in ultraviolet receptors of Limulus. J. Gen. Physiol., 59: 167-185.

Rosenfeld, T., Alchalal, A., and Ottolenghi, M. (1972) Nanosecond laser photolysis of rhodopsin in solution. Nature, 240: 482-483.

Suzuki, T., Sugahara, M., and Kito, Y. (1972) An intermediate in the photoregeneration of squid rhodopsin. Biochim. Biophys. Acta, 275: 260-270.

Yoshizawa, T. (1972) The behaviour of visual pigments at low temperatures. In Handbook of Sensory Physiology, ed. by Dartnall, H. J. A. Springer-Verlag, Berlin, Heiderberg, and New York, Vol. 7/1, pp. 146-179.

Yoshizawa, T. and Wald, G. (1964) Transformations of squid rhodopsin at low temperatures. Nature, 201: 340-345. 